\title{
Difficile réconciliation en France entre logique de rentabilité du secteur médico-social et démarche éthique souhaitée par les patients
}

\author{
Odile Grandjean-Rucar ${ }^{\mathrm{a}}$
}

RÉSUMÉ. Les lois françaises de 1982 à 2016 ont élaboré un cadre contraignant pour le secteur médico-social, également incité à se comporter de façon éthique et responsable. Deux logiques se sont affrontées : rentabilité versus recherche de satisfaction des patients. La divergence entre les objectifs des soignants et des gestionnaires s'est d'autant plus amplifiée que les usagers contestent la légitimité des dépenses publiques. Les innovations technologiques favorisent la connaissance des attentes des patients. Le processus devrait être ascendant (des besoins et attentes des patients vers la direction), il est en réalité souvent descendant (des exigences de la direction vers les patients). La réforme de 2018 réintègre la qualité de l'organisation territoriale des soins, mais devra répondre aux exigences d'équilibre, de sécurité, et d'éthique. De nombreux cas vécus par des patients illustrent ces incohérences : la situation semble critique, mais des réponses organisationnelles peuvent être données à défaut de solutions plus onéreuses immédiates.

\begin{abstract}
The French laws between 1982 and 2016 bave created a compelling environment in the medical and social areas, which are actually expected to behave in an ethical and responsible way. Two hardly reconcilable ideas have come to face each other: a profit-oriented one and a patient satisfaction-oriented one. Thus, it has emphasized the divergence between caregivers and administrators' goals. Moreover, many users are questioning the public expenses legitimacy. Besides, technological innovations (informatics, databases, interconnecting files, datamining...) have permitted to measure and acknowledge patients' expectations more precisely (Medical information departments in hospitals, satisfaction surveys...). We should observe an ascending process (from patients' needs and expectations to management), yet it is often the opposite (from management demands on patients), which works against organizational ethics. A reform announced in July 2018, which focuses on territorial healthcare organizations, seems promising, yet ambitious regarding the means that will be dedicated to it. However, it remains to be seen if mobilization of territorial resources will be balanced, safe, and ethical. Lots of real cases that patients have come through show a lack. of consistency, leading to a troubling situation for both patients and their families: the situation seems critical, but organizational solutions can be provided, in the absence of more expensive and immediate solutions.
\end{abstract}

\section{Introduction}

Depuis plus de 30 ans maintenant, la France en général et le secteur médico-social en particulier ont connu de nombreuses réformes et mutations qui se sont inscrites dans un cadre multiforme : la Révision Générale de Politiques Publiques $(2007)^{1}$, un déficit budgétaire chronique et un déséquilibre des comptes de la Santé, la réforme des 35 heures (2002) appliquée au secteur hospitalier sans contrepartie financière ${ }^{2}$, la loi portant réforme de l'Hôpital et relative aux Patients, à la Santé et aux Territoires adoptée en 2009 (ci-après désignée loi HPST) ${ }^{3}$. Cette réforme de 2009 est majeure et marque un virage historique : elle vise la modernisation des établissements de santé, l'accès de tous à des soins de qualité, la prévention et la santé publique, ainsi que l'organisation territoriale du système de santé.

À travers ces réformes, la volonté des différents gouvernements de moderniser le système de santé s'est accompagnée d'un regroupement des structures, ce

a Agrégée d'économie et de gestion, Université de Lorraine, directrice adjointe de l'IUT de Metz 
qui a entrainé la fermeture des plus petites unités dans le but d'assurer aux patients des soins de qualité en toute sécurité. Ainsi, sous l'effet des réorganisations et restructurations liées aux différentes réformes, le nombre d'entités géographiques de statut public ou privé à but lucratif a diminué. Ces mouvements tiennent à de nombreux facteurs liés aussi bien à la rationalisation de la prise en charge qu'à l'amélioration de sa qualité. L’idée dominante étant que plus un établissement pratiquait d'actes du même type, plus il était spécialisé et performant, même si cela signifiait également un maillage moins serré dans les zones à faible densité de population, surtout celle en régions. À ces objectifs de réorganisation du système santé par la loi HSPT s'est ajouté le principe de déconcentrer l'action de l'État en s'inspirant du privé, notamment en termes de gouvernance, mais sous l'égide d'un outil de gestion publique : les Contrats Pluriannuels d'Objectifs et de Moyens (ci-après appelés les $\mathrm{CPOM}^{4}$ ).

Modifier le type de gouvernance, même avec des objectifs louables, a eu des conséquences sur les patients. D'ailleurs, ces derniers sont-ils devenus des usagers, voire des consommateurs? Autant de terminologies avec des sens bien précis et des implications différentes. Les patients reçoivent des soins ou des attentions médicales, les usagers utilisent des biens ou des services publics (non marchands) pour satisfaire un besoin d'intérêt général et les consommateurs achètent des biens et services marchands pour satisfaire un besoin.

Par ailleurs, en principe, les innovations technologiques continues (liées aux NTIC) doivent permettre une meilleure connaissance des besoins et des attentes des patients, des pratiques et être au cœur des CPOM. Nous devrions avoir une approche reposant sur un processus ascendant (des patients vers la direction). Dans les faits, on observe, en raison des nombreuses contraintes (réglementaires et budgétaires) exactement l'inverse, soit un processus descendant (des besoins de la direction qui établit les besoins et les budgets vers les patients).

Le vaste domaine de la santé publique se doit d'être éthique: des traditionnelles distinctions entre morale et éthique, nous retiendrons en tant que gestionnaire, celle de certains philosophes (E. Levinas, P. Ricoeur) voyant la morale comme un ensemble de devoirs commandant de faire le Bien et l'Éthique comme une réalisation raisonnable des désirs : l'éthique apparait dès lors comme un cadre de réflexion permettant d'améliorer le réel par une attitude raisonnable, responsable et respectueuse.

Dès lors, face à ces nombreux bouleversements, on peut se demander comment il est possible d'instaurer des pratiques éthiques dans les hôpitaux, remettant l'humain au cœur du processus et prenant en considération les contraintes réglementaires et budgétaires. Ainsi allons-nous commencer par analyser l'inversion du processus de gestion qui aboutit à une déshumanisation des pratiques et donc ébranle l'approche éthique. Ce questionnement devrait déboucher ensuite sur des pistes de réflexion et des propositions pour une gouvernance plus responsable et respectueuse ${ }^{5}$.

\section{Comment l'inversion du processus de gestion ébranle l'éthique}

\section{1 Éthique et hôpital : principes}

Retenons cette définition de Marc Horwitz (2009), journaliste spécialiste des questions de santé publique, selon laquelle l'éthique est un point d'équilibre entre ce qui différencie « le soigner, le guérir » du "prendre soin, prendre attention » de la part des équipes soignantes. C'est aussi, selon Olivier Toma (2012), directeur de plusieurs établissements de santé et spécialisé dans le développement durable en santé, placer non pas seulement le malade ou l'assuré, mais l'homme au sein des pensées et pratiques, comme les dispositifs de soin. De son point de vue, il ne faut pas uniquement organiser un monde de réponse à la maladie, mais il faut surtout promouvoir un monde de la santé.

Ainsí l'éthique doit garantir le droit à la santé pour tous (droit à une information complète et compréhensible, à son dossier médical), l'égalité d'accès aux soins, le respect de la dignité humaine. Des questions fondamentales relatives au système actuel émergent et en voici des exemples :

- Avons-nous le libre choix des soignants, des établissements?

- Y a-t-il une disparité dans l'offre de soins?

- La faible densité médicale dans certaines régions est-elle source d'inégalités?

- Le reste à charge (part de dépense qui reste à la charge de l'assuré après remboursement de l'assurance-maladie obligatoire, des complémentaires santé) remet-il en cause l'égalité d'accès aux soins?

- Le système français est-il à deux vitesses?

- Comment la douleur est-elle prise en charge? 
Toutefois, on ne peut négliger ces deux questions fondamentales : les impératifs économiques ne l'emportent-ils pas parfois sur les principes éthiques? Faut-il limiter les soins aux personnes âgées au profit de personnes plus jeunes, donc plus productives? Ces personnes âgées sont nommées littéralement les «bloqueurs de lits» chez les Anglo-saxons. Question qui devrait être dérangeante, même si l'on est gestionnaire, mais qui, comme nous le verrons un peu plus loin, commence à se poser réellement. Et n'étonne plus systématiquement. Or, limiter les soins en tenant compte de lâge est discriminatoire, donc contraire à l'éthique, aux lois sur la discrimination, notamment aux chartes des droits et des libertés des personnes.

Au fil des réformes s'est amplifiée la contradiction majeure entre le service rendu par l'État à l'humain et son financement au moyen de la dépense publique. D'autant plus que la redistribution semble de moins en moins légitime aux yeux de la population.

On comprend aisément les orientations affichées de notre politique de santé moderne : il s'agit de favoriser la qualité de la vie par rapport à la durée (donc il faut mettre en place une vraie politique de prévention), lutter contre les morts prématurées, permettre à tous d'avoir une fin de vie apaisée et de mourir dans la dignité. Cette mission est respectable, à condition de ne pas avoir à faire des choix déchirants au nom d'impératifs économiques. Et cela signifierait que nous avons une vision complète et juste de la réalité des pratiques, point sur lequel nous reviendrons un peu plus loin.

\section{2 État des lieux : cadre réglementaire et budgétaire, chiffres et contexte}

- La loi no 2002-2 du 2 janvier 2002 rénovant l'action sociale et médico-sociale (ci-après désignée la loi de 2002) établit la notion juridique de droit des malades et permet de mieux représenter les usagers dans les grandes institutions de la santé et les hôpitaux. Elle donne le droit aux patients d'accéder à l'intégralité de leur dossier médical. Pour information, le dossier médical personnel (DMP)créé par la loi n 2004-810 du 13 août 2004 relative à l'assurance maladie (ci-après désignée la loi de 2004), a été rebaptisé et transformé en Dossier Médical Partagé en 2015 (il est informatisé). Mais la loi n 2016-41 du 26 janvier 2016 sur la modernisation de notre système de santé (ci-après désignée la loi de
2016) prévoit un déploiement progressif sur le territoire. La loi de 2002 permet aussi une indemnisation de l'aléa thérapeutique (l'accident médical, l'affection iatrogène, l'infection nosocomiale), modifie les dispositions relatives à l'hospitalisation sans consentement et oblige les médecins à faire connaître leurs liens avec les entreprises quand ils communiquent au sujet d'un produit médical.

- Dans le Plan Hôpital de 20077, la tarification à l'activité est un élément central de la nouvelle Gouvernance Hospitalière mise en place en 2005. Elle est conforme à l'esprit de la LOLF (Loi organique no 2001-692 du 1er août 2001 relative aux lois de finances) imposant une logique de résultats, alors qu'auparavant le financement basé sur une Dotation Globale de Fonctionnement reposait sur une logique de moyens. On a donc assisté au désengagement de l'État face à la montée de l'individualisme.

- La loi HPST de 2009 a pour objectif de moderniser et de réorganiser le système de santé. Elle renforce le pouvoir des chefs d'établissements, permet de mettre en commun des moyens entre établissements autour d'un centre de référence, cherche à améliorer la répartition des médecins sur le territoire, prévoit des dispositifs pour développer l'éducation thérapeutique des patients, crée les Agences Régionales de Santé (ARS). Ces dernières mettent en œuvre les dispositifs prévus par la loi sur le plan régional.

- L'indice ICALIN (Indicateur Composite des Activités de Lutte contre les Infections Nosocomiales) créé en 2005 sous la pression des associations de patients et dans le cadre d'une politique de prévention, même si cet indicateur a des limites réelles.

- La Charte de la personne hospitalisée mise en place par la circulaire de 2006 dans le cadre de la loi de 2002 garantit au patient la liberté de choix de l'établissement, l'accès à des soins et traitements de qualité, la possibilité d'obtenir une information loyale et accessible, la nécessité au moment de l'acte médical de recueillir le consentement éclairé des patients. 


\subsubsection{Quelques chiffres permettant de compléter cet état des lieux (Tableaux de l'Économie Française ${ }^{8}$ )}

En 2014, les dépenses de santé représentent $12 \%$ du PIB de la France, soit 257 milliards d'euros, un des taux les plus élevés parmi les pays de l'OCDE (10 \% pour le Canada). La consommation de soins et biens médicaux (CSBM) est évaluée à 190 milliards d'euros, soit environ 2900 par habitant. Le reste à charge des ménages s'élève à 16,2 milliards d'euros, soit 8,5 \% de la CSBM. La France se classe $\sigma^{e}$ parmi les pays de l'OCDE pour les dépenses courantes de santé en $\%$ du PIB. Le nombre de bénéficiaires de la $\mathrm{CMU}^{9}$ (Couverture Maladie Universelle) est de 5 millions de personnes, chiffre en augmentation, car le gouvernement a élargi les conditions d'accès à ce dispositif pour lutter contre la pauvreté (le seuil de pauvreté est égal à $50 \%$ du revenu médian, soit environ 860 euros par mois).

Ces chiffres montrent que l'État dépense des sommes considérables pour la santé, mais que cela ne signifie pas pour autant que ces dépenses soient efficaces et inscrites dans une stratégie de prévention et de prise en charge à long terme. Ainsi, l'augmentation de l'espérance de vie de la population entraine une modification des pathologies, notamment celles liées au vieillissement. D'autre part, l'amélioration continue des soins, très positive pour la population a un coût. Les déserts médicaux sont source d'inégalités fortes pour l'accès aux soins. Le ministère de la Santé définit le désert médical comme étant un territoire dont la densité de médecins (ratio rapportant les effectifs de médecins, omnipraticiens et spécialistes, à la population d'un territoire donné) est inférieure de $30 \%$ à la moyenne nationale. L'INSEE (Institut National de la Statistique et des Études Économiques) estime à 337 le nombre de médecins en France pour 100000 habitants en 2017; ainsi le désert médical concerne les zones géographiques (le plus souvent des départements ruraux) dans lesquelles la densité de médecins est inférieure à 235. Le désert médical se caractérise par une offre médicale insuffisante sur la zone pour répondre aux besoins de la population, et qui est due principalement à une mauvaise répartition ${ }^{10}$ des praticiens.

La féminisation de la profession médicale implique aussi davantage un exercice de la médecine à temps partiel, ce qui en soi ne devrait pas poser question, mais qui devient problématique eu égard au numerus clausus (le nombre de places mises au concours pour entrer en $2^{\mathrm{e}}$ année de médecine). Ajoutons que sur 15 millions de personnes aux urgences, 10 millions pourraient être prises en charge par la médecine ambulatoire : sont mis en cause principalement la gratuité des soins aux urgences, le fonctionnement de la médecine de garde, le grand nombre de personnes âgées dont on ne sait que faire.

\subsection{Caractérisation des pratiques : résultats des entretiens}

Concernant le profil des répondants, ils ont une formation universitaire $(29 \%)$, technique $(39 \%)$ et autres $(32 \%)$. Il en ressort que les transformations du système de santé ont des conséquences antinomiques : si elles permettent de mieux soigner avec une meilleure sécurité (exemple des plateaux hospitaliers), elles n'en induisent pas moins des pratiques parfois contraires à l'intérêt et au respect des patients et même à la dignité humaine dans certains cas.

Les exemples de pratiques et le recueil de témoignages (patients, soignants, journalistes) qui suivent montrent des dysfonctionnements majeurs liés au fonctionnement des hôpitaux et aux autres acteurs du secteur médico-social: EHPAD (Établissement d'Hébergement pour Personnes Âgées Dépendantes), SSR (Soins de Suite et Réadaptation).

Tout d'abord, malgré le droit à l'information, le patient et ses proches n'obtiennent pas toujours des explications claires et complètes sur la pathologie, les traitements, la surveillance ultérieure à mettre en place. Plusieurs facteurs sont en cause, soit le manque de temps des médecins, la difficulté pour les infirmières à donner des éléments médicaux sans l'aval de ces derniers, la carence en formation spécifique des soignants, et parfois le faible intérêt de certains médecins pour la psychologie et les relations humaines.

Outre les innovations technologiques, le système d'information ${ }^{11}$ (SI) n'est pas toujours performant par manque de compatibilité entre les différents matériels et logiciels (d'où des interconnexions problématiques), et par manque de temps et de compétence du personnel pour en utiliser toutes les fonctionnalités. Ceci se traduit dans certaines structures par une mauvaise transmission d'informations médicales entre les services (de la réanimation 
à la gériatrie par exemple), donc une méconnaissance du dossier du patient. Parfois, le patient hospitalisé suit un traitement lourd avant hospitalisation dont les médecins de l'hôpital n'ont pas connaissance, car ils n'ont pas eu le dossier mis à jour par le médecin traitant quand il y a une urgence. Dans certains cas, ce sont les familles elles-mêmes qui résument le dossier médical dans les services pour gagner du temps. Elles amènent la prescription du médecin traitant, et la donnent directement aux infirmières. De plus, en dépit des obligations législatives et réglementaires, certains médecins traitants demeurent en désaccord lorsque vient le temps de remettre le dossier médical à un patient qui désire changer de praticien.

On constate donc que, malgré la modernité des processus et des outils informatiques, la coordination est inefficace parce que la procédure relative au dossier informatisé du patient n'est pas au point et que le lien immédiat entre médecin traitant et hôpital en cas d'urgence est inopérant.

Avec le changement de logique induit par les lois de 2002 et 2009, le nombre de lits en service de réanimation est parfois insuffisant. Dans certaines situations de crise, les chefs de service sont amenés à faire des choix entre les patients en s'aidant des probabilités de survie. Et parfois désemparés, ils le disent brutalement aux familles qui ont ainsi le sentiment désastreux qu'une personne âgée doit laisser la place à un patient plus jeune. La question ainsi posée est intolérable, car il n'est pas admissible qu'on ait à faire un tel choix.

Selon différentes sources ministérielles et études ${ }^{12}$, la prévalence de personnes âgées de plus de 75 ans placées en institutions (EHPAD), dénutries ou à risque de malnutrition approche les $60 \%$ de la population étudiée. Une des principales raisons en est l'anorexie. Il importe alors qu'en cas d'hospitalisation, le personnel soit vigilant quant à la composition des repas. Malgré les contraintes de coût compréhensibles, il faudrait surtout servir une alimentation équilibrée, tenant compte des pathologies, l'impératif de qualité de l'alimentation dépassant celui de quantité. La plupart du temps, les repas sont identiques pour la jeune maman et la personne âgée, alors que les besoins diffèrent fondamentalement. Que dire des plateaux-repas préparés pour ceux qui ont des colopathies graves, des allergies au gluten, avec des aliments contre- indiqués pour leur état de santé? Le personnel qui sert les repas n'est pas toujours informé de ces particularités diététiques et lorsque c'est le cas, parce qu'il n'y a pas de solution simple immédiate, vous suggère d'apporter ce qui manque pour compenser.

Par ailleurs, la restauration est souvent soustraitée à des entreprises qui ont des actionnaires ayant une optique de rendement à court terme. Il serait nécessaire que les directions des établissements leur fassent signer des chartes, des engagements et redéfinissent les règles du jeu. Comment accepter l'idée qu'une entreprise puisse faire du bénéfice au détriment de la qualité des repas, qui inclut la diversité alimentaire, la recherche de saveurs, l'alimentation plaisir, tout en ayant en mémoire la perte d'appétit des personnes institutionnalisées? Bien souvent, les familles apprennent par hasard qu'elles peuvent rencontrer des nutritionnistes pour les aider au suivi alimentaire des proches à la sortie de l'hôpital. Cela devrait être systématiquement proposé. Dans les établissements, hôpitaux et EHPAD, la plupart du temps il y a peu de cohérence entre pathologies, état de santé général, besoins nutritionnels et repas servis.

D'ailleurs, en SSR, il y a parfois aussi une mauvaise gestion des médicaments, et les patients attendent parfois plusieurs jours pour obtenir leur traitement. On rapporte qu'une patiente en SSR qui a subi une arthroplastie de la hanche, et qui était atteinte de troubles intestinaux chroniques, a dû attendre plusieurs jours pour se voir administrer la médication appropriée, parce que ce n'est pas le traitement principal lié à la prise en charge.

Dans les hôpitaux, les SSR et les EHPAD soumis à la tarification à l'activité, le temps prévu pour la toilette d'une personne alitée est très court. De plus, certains personnels comme les aides-soignants (et c'est un métier très dur avec peu d'espoir de promotion et une rémunération peu motivante) n'ont pas toujours la vocation ni les qualités psychologiques adaptées à ce travail. Les soins apportés sont parfois à la limite de la brutalité (qui peut être aussi verbale) et de l'atteinte à la dignité. Ces sujets sont difficiles à aborder, mais des personnes âgées témoignent qu'elles ne se sentent pas respectées (particulièrement dans les cas d'incontinence). On met aussi systématiquement des couches aux patients quand ils peuvent aller aux toilettes avec une aide, parce que c'est plus simple à gérer. Une 
telle pratique porte aussi gravement atteinte au respect de la personne. C’est un sujet méconnu du grand public, mais très sensible dans les EHPAD et SSR.

Les médecins sont parfois récalcitrants quand il faut déterminer le lieu du SSR qui suivra l'hospitalisation : une personne hospitalisée en urgence par exemple à Épinal (au cœur des Vosges) se voit imposer un SSR dans ce département, alors que la famille souhaite un SSR plus proche, en Meurtheet-Moselle (en Lorraine), pour faciliter les visites. Pourtant, l'appui des proches est essentiel pour le soutien psychologique du patient. Il faudra lutter contre le médecin pour avoir gain de cause, car la procédure prend trop souvent le pas sur le confort du malade.

Beaucoup de personnes âgées seraient mal soignées si les proches n'étaient pas là pour questionner, alarmer, informer. Il n'y a pas forcément d'intentions malveillantes des soignants, mais plutôt le signe d'un mauvais fonctionnement du système et de dérives, dont les soignants sont aussi victimes.

Ces témoignages, s'ils ne permettent pas de généraliser, montrent que certaines pratiques dans le secteur médico-social sont questionnables sur le plan éthique et que le processus est inversé : les objectifs de la direction s'imposent pour des raisons réglementaires, financières aux patients dont les attentes ne sont pas forcément respectées. Les acteurs des organisations du secteur médico-social dans lesquelles la marchandisation (finalité orientée vers la rentabilité) s'est infiltrée ont a priori des attentes et des logiques d'action difficilement conciliables. Peut-on en dépit de cela instaurer des pratiques éthiques?

\section{Quelles sont les pistes de réflexion et les propositions pour une gouvernance plus responsable et respectueuse?}

Réintroduire du lien qui donne sens dans la relation soignant/patient est un gage d'éthique. Les outils mis en place (Système d'Information (SI) et Département d'Information Médicale (DIM), gestion des risques, certifications, etc.) pourraient favoriser la mise en place d'autres indicateurs d'une « qualité » à redéfinir par l'ensemble des acteurs.

\subsection{Gouvernance}

Les parties prenantes sont les professionnels de la santé (médecins, autres soignants), la direction, les cadres de santé, les salariés, les fournisseurs, les sous-traitants et les patients, acteurs d'une organisation qui se trouvent concernés par les décisions de l'organisation.

\section{Administratifs}

L'application de loi HPST pose la question de la double hiérarchie des administratifs et des soignants, et donc d'une dualité de vision entre politiques - technocrates et professionnels de la santé. Le directeur de l'établissement a un pouvoir fort de gestionnaire, cependant limité par le fait qu'il soit désigné par le directeur général de l'ARS (Agence Régionale de Santé). Nommé en conseil des ministres, il est en quelque sorte un préfet de la santé doté d'un pouvoir énorme. Le risque est que les établissements soient de plus en plus gérés comme des entreprises. De plus, les ARS pourront contractualiser des directeurs d'hôpital venus du privé et qui ne seront plus formés par l'École des hautes études en Santé13. Les conséquences possibles sont des différences de rémunération entre directeurs et un mode de management plus tourné vers la rentabilité (exemple d'objectif: diminuer la masse salariale).

De façon plus globale, la direction générale de la Santé s'appuie sur de nombreuses agences nationales auxquelles est alloué un budget (ANSM, Agence Nationale de Sécurité du Médicament; ANSES, Agence Nationale de Sécurité sanitaire de l'alimentation, de l'Environnement et du travail; ANAP, Agence Nationale d'Appui à la Performance,... plus 26 ARS, Agences Régionales de Santé). Beaucoup de moyens (80\% du budget assuré par les subventions de l'État et $20 \%$ par l'assurance maladie) et d'énergie pour des résultats limités avec des redondances, pour une efficacité qui n'est pas toujours démontrée (sur cette analyse, les avis divergent) (Horwitz, 2009; Toma, 2012). Le financement est complexe, inégalitaire (en raison d'une disparité de subventions régionales) et parfois peu transparent.

La mise en place de CPOM signés entre l'établissement et les ARS constitue un outil d'incitation, d'évaluation, de contrôle et de financement permettant en théorie d'atteindre les objectifs fixés. Mais cet outil a parfois été détourné de sa finalité et 
utilisé comme un moyen d'autorité, de discrimination et de pouvoir (Toma, 2012), les moyens nécessaires à la réalisation des objectifs n'étant pas toujours attribués. Ajoutons que si le CPOM n'est pas signé, l'établissement risque d'être déconventionné; on comprend finalement qu'un schéma coercitif s'est mis en place, les ARS œuvrant pour réduire le déficit de l'assurance maladie et pas forcément pour améliorer la santé publique.

\section{$\underline{\text { Propositions }}$}

- Réinventer la gouvernance hospitalière en s'assurant que les intérêts financiers ne priment pas sur la responsabilisation des soignants et sur le respect de la dignité des patients.

- Créer une grande Agence serait nécessaire, en établissant des contre-pouvoirs plus efficaces et tournés vers le patient. Cela relève de la volonté de l'État.

Soignants

Quelques propositions issues des réflexions préalables et divers travaux méritent d'être présentés :

La formation des médecins pourrait être revue, d'abord en changeant le mode de sélection à l'issue de la 1 ère année (QCM : utilisation à cet égard de Questionnaires à Choix Multiples) laquelle favorise les étudiants normés sur un plan scientifique et dont la motivation à soigner n'est pas vérifiée.

La formation devrait aussi faire plus de place à la psychologie, parent pauvre du programme; à l'apprentissage de l'anglais, grand oublié, indispensable pour comprendre les revues internationales de recherche scientifique; à une culture statistique plus solide quand on sait qu'à partir de trois médicaments, on induit des interactions qu'on ne maitrisera pas forcément et qui produiront des effets indésirables majeurs.

Ces pistes de solution ne sont pas forcément coûteuses, mais nécessitent de l'énergie de la part des décideurs et entreraient dans une perspective éthique privilégiant le patient.

\section{Lespatients}

Il serait souhaitable que les patients deviennent de vrais acteurs de leur santé et soient responsabilisés.
Le système de santé est encore considéré comme gratuit par plusieurs d'entre eux. Les mesures instaurées depuis quelques années ont augmenté le reste à charge, créant parfois une médecine à deux vitesses, sans pour autant déclencher une vraie prise de conscience par tous. Depuis les années 90, les patients sont devenus consuméristes et les magazines publient régulièrement des classements d'établissements du secteur par type de pathologie. Se pose la question de l'objectivité de tels indicateurs.

\section{$\underline{\text { Propositions }}$}

- Rendre le patient acteur de sa santé, en développant le nombre d'infirmièresconseils, qui accompagneraient les patients avant et après l'hospitalisation, pour expliquer les précautions à prendre et ainsi faire de la prévention, (par exemple sur le rôle du tabac dans la cicatrisation). Ceci pourrait aussi être fait plus systématiquement sur l'hygiène de vie en général (alimentation, exercice...).

- Impliquer les associations d'usagers pour qu'elles ne soient pas limitées à un rôle de revendication.

\subsection{Système d'Information (SI) et Département d'Information Médicale (DIM)}

Le DIM, généralisé en 1989, est chargé de « la mise en œuvre des traitements automatisés des données médicales nominatives ». Il a en charge la collecte, le recueil et le traitement des données médicales qui permettent ensuite de déterminer la partie des financements qui est liée à l'activité. Ce département est impliqué dans le pilotage stratégique et financier de l'établissement (Toma, 2012).

Au-delà de ce travail, le système d'information médical aboutit au calcul d'indicateurs de qualité des soins. Mais la façon dont ces indicateurs sont calculés pose problème parfois, parce qu'elle relève d'une conception instrumentaliste de l'organisation opposant objectifs et finalités du système. À ce titre, on peut citer le débat actuel autour de la pharmacovigilance (affaires MEDIATOR ${ }^{14}$ et DOCETAXEL ${ }^{15}$ ) alors que les médecins sont censés déclarer les effets indésirables de médicaments, afin d'assurer la sécurité 
des patients. Parfois, ils hésitent à le faire pour ne pas se rendre responsables des conséquences financières. De même, une pression est exercée sur les médecins pour limiter cette déclaration sous l'impulsion de responsables de l'État et de lobbys pharmaceutiques.

Dans le même esprit, on peut noter que les traitements de données médicales doivent en principe apporter une meilleure connaissance des maladies nosocomiales et iatrogènes. Mais l'approche instrumentaliste néglige parfois cette voie, pourtant dans l'intérêt des patients et de la santé publique. Or, ces analyses devraient permettre une évaluation des pratiques, un calcul des coûts plus pertinent et complet.

\section{$\underline{\text { Propositions }}$}

Dans un souci éthique (sauver des vies) et à moindre coût (le système existe déjà), il serait pertinent d'effectuer des études statistiques (pas seulement descriptives) sur les conséquences de l'hyperconsommation médicale (personnes âgées ayant plusieurs pathologies lourdes), sur les maladies iatrogènes (par exemple sur les problèmes liés aux excipients des médicaments génériques), sur la traçabilité des dispositifs médicaux (des prothèses de hanche non conformes ont été posées à des patients entre 2003 et 2010 à la suite d'une réglementation plus souple; d'où un risque d'effets indésirables, comme le rappelle l'affaire des prothèses mammaires de la société PIP, en 2013).

\subsection{Qualité et prévention}

Il suffit de rechercher sur les sites des établissements médico-sociaux pour trouver leur organigramme, leur démarche qualité et leur participation à la politique de prévention. Bien souvent l'organigramme révèle une confusion entre des instances d'exécution et des instances de contrôle ou d'évaluation, ce qui ne garantit pas l'indépendance nécessaire. Ainsi en est-il du pôle Ressources et Qualité dans certains établissements. Or, l'absence de clarté d'un organigramme peut laisser penser qu'il y a collusion d'intérêts. De même, certains établissements ne communiquent qu'un organigramme administratif, sans la partie médicale. Ce sont des pratiques qui remettent en question le respect de la clientèle, alors que le DIM et le SI ont été instaurés pour améliorer la qualité des soins.
La démarche qualité n'est pas clairement identifiable sur tous les sites d'établissements. Parfois, on y trouve surtout des formulations très générales, peu concrètes et peu impliquantes. On est loin de l'esprit lean d'amélioration continue officiel. Le concept est né dans l'industrie pour satisfaire des clients, donc des entreprises à but lucratif. Or, il a été transposé sans le moindre aménagement dans les établissements de soins, pour lesquels la finalité première n'est pas le bénéfice. De façon générale, les actions de prévention efficaces à long terme ont été négligées par certains établissements, alors que des économies de temps et d'argent pourraient en résulter.

\section{Quelques pistes}

L'éducation thérapeutique du patient dont on a traité plus haut est indispensable, car les patients sont aussi ses facteurs de la dégradation du système. Il faudrait généraliser ces pratiques éducatives.

- Protéger les lanceurs d'alerte contre les mesures de représailles et les poursuites judiciaires semble indispensable (ex. cas de l'amiante et du bisphénol). Une loi est présentement en cours d'élaboration en France;

- Intégrer au système de santé traditionnel les médecines alternatives moins coûteuses et complémentaires (plus d'accompagnement des patients). Cela pourrait se généraliser comme on l'observe déjà à certains endroits (en France ou en Australie). Mais il faut encadrer ces dispositifs pour prévenir les dérives;

- Réfléchir à la mondialisation médicale responsable est une autre piste (tourisme médical certifié et encadré) à la condition de ne pas le faire au détriment des assurés français et d'en faire une source de rentabilité supplémentaire. Car depuis 10 ans, l'État impose des solutions souvent malthusiennes (réduction du nombre de lits).

Depuis le début de 2018, en France, des études menées par des institutions (dont le Sénat), des alertes et reportages sur des scandales (maltraitance avérée dans des EHPAD...) et des dysfonctionnements majeurs (personnel soignant insuffisant...), ont révélé les failles du modèle de fonctionnement des établissements de santé, commencé à envahir les médias et à générer un questionnement des élus, responsables, et citoyens. 
On peut noter de façon générale une évolution de la société impliquant dans un contexte de vieillissement de la population, des changements d'attitudes et de comportements; ceux-ci remettent en cause des modèles qui montrent clairement leurs limites, dans lesquels la rentabilité prime souvent sur la qualité et l'éthique. Les différents protagonistes se préoccupent de remettre l'humain au cœur des différents dispositifs, dans un contexte de réorganisation du territoire.

Cette prise de conscience semble collective et urgente, à tel point que le gouvernement a annoncé en juillet 2018 une réforme en profondeur du système de santé, qui sera élaborée à partir des recommandations d'un groupe de travail réunissant les différents acteurs. Les objectifs annoncés de cette réforme visent la qualité et la pertinence des soins, la formation continue, la qualité de vie au travail des professionnels de santé, les modes de financement et de régulation, l'organisation territoriale des soins.

Les études de santé seront également réformées et le numerus clausus supprimé, afin de lutter contre la désertification médicale, la sélection (sur le profil également) et la formation (en psychologie, par exemple) des futurs médecins. Le gouvernement conscient des limites du gigantisme des organisations, né des précédentes réformes, annonce clairement réfléchir à renforcer, voire rétablir un maillage territorial équilibré, gage de proximité et qualité. Ceci se fera en maintenant au même niveau le budget de la santé, alors qu'une diminution était initialement prévue. L'État dit se préoccuper d'intégrer une exigence éthique dans sa réforme en tentant de concilier coût - efficacité - service de qualité et de proximité. Il entame une réflexion sur le lien ville hôpital, et annonce vouloir labelliser environ 600 structures de proximité d'ici fin 2022. Bien sûr, il existe déjà des initiatives intéressantes dans ce domaine, par exemple les pôles et maisons de santé, les plateformes hospitalières, etc.

On peut faire un parallèle avec la grande distribution. Le développement des hypermarchés dans les années $70-80$ a favorisé leur concentration au détriment des petits commerces de proximité. Dans un premier temps, le consommateur y a gagné en termes de choix et de prix. Mais depuis quelques années, les ménages cherchent toutefois à remettre du lien dans leurs actes de consomma- tion : produits locaux, relation proche avec le vendeur... On observe une remise en cause du généraliste de grande taille qui a quasiment éliminé le commerce de détail indépendant. D’où une désorganisation du territoire entraînant des déséquilibres locaux.

Ce même phénomène de centralisation a aussi affecté le domaine de la santé. Il a certes permis de créer des pôles d'excellence concentrés dans des établissements de grande taille en supprimant les petites unités peu rentables et peu performantes, mais il a aussi induit de profonds déséquilibres en ce qui concerne l'égalité d'accès aux soins des citoyens (principe fondateur de l'État) et la dimension humaine de la relation soignant - patient dans bien des cas. On peut noter les limites suivantes: dépersonnalisation du service rendu, lourdeur des processus, déshumanisation, perte de repères, uniformisation des pratiques, bref, une désorganisation du territoire que ce projet de réforme vise à corriger.

Il n'est pas envisagé de réimplanter des hôpitaux dans les petites villes, mais peut-être faut-il développer les pratiques de plateformes médicales entre grandes villes avec des CHR (Centres Hospitaliers Régionaux) et moyennes villes de 40000 à 50000 habitants : déplacements de certains spécialistes dans des domaines pointus, venant opérer un jour ou deux par semaine dans la moyenne ville; accès rapide et facilité au matériel médical de pointe pour les patients de la moyenne ville; échange de bonnes pratiques codifié, formalisé et capitalisé...

L'implantation de maisons médicales regroupant médecins, infirmiers, orthophonistes, kinésithérapeutes, pourrait être étendue de façon rationnelle et équilibrée à tout le territoire, à la condition d'organiser un transfert rapide et systématique des patients par hélicoptère vers le grand centre hospitalier le plus proche en cas d'urgence. Ainsi, on rétablirait un maillage du territoire plus complet et dense dans de bonnes conditions de sécurité, ce qui répond à l'exigence éthique de la relation patient/soignant.

Le développement de la télémédecine pourrait s'accélérer, mais en veillant à maintenir un contact humain professionnel et respectueux entre le médecin à distance et le patient. Il convient de veiller à garantir à tous le service de proximité, l'éthique de la relation, l'égalité d'accès aux soins, ce qu'un maillage étroit, dense et équilibré du territoire favoriserait. 


\section{Conclusion}

En conclusion, remettre l'éthique au cœur du système de santé est possible et parmi les pistes et propositions évoquées, n'est pas toujours onéreux; mais cela exige de changer la vision de l'Organisation dans le monde médico-social. Certaines propositions peuvent être mises en place directement au sein des établissements, car elles ne nécessitent pas de moyens financiers importants; d'autres rendent indispensable un changement de conception au plus haut niveau de l'État. Toutes impliquent l'ensemble des acteurs.

\section{NOTES}

1 RGPP : réforme visant à diminuer les dépenses publiques tout en renforçant l'efficacité et la qualité de l'action publique.

2 La réforme des 35 heures par semaine n’a pas été accompagnée du nombre d'embauches prévues, faute de moyens et dans une moindre mesure de candidats, dans un contexte de travail du personnel $24 \mathrm{~h} / 24$.

3 Loi HSPT comprend 4 volets : l'hôpital, la répartition des médecins et l'accès aux soins de villes, les mesures de santé publique et la prévention, enfin la création des Agences régionales de santé (ARS) chargées de coordonner dans un cadre territorial l'ensemble des politiques de santé (hôpital, médecine de ville, santé publique et prévention).

4 Un CPOM est un contrat par lequel un organisme gestionnaire d'établissements, de services sociaux ou médico-sociaux, s'engage auprès d'une autorité de tarification sur une période pluriannuelle pour, en fonction des objectifs d'activité poursuivis par ses établissements, bénéficier d'allocations budgétaires correspondantes. Pour plus d'information sur les Contrats Pluriannuels d'Objectifs et de Moyens, on consultera le site suivant : https://solidarites-sante.gouv.fr/IMG/pdf/Presentation_CPOM.pdf

5 Cette étude s'appuie sur des entretiens libres menés auprès de 8 patients ayant subi plusieurs hospitalisations pendant plusieurs années, de 20 soignants et sur l'analyse de 12 articles et reportages. Une région administrative a été retenue, soit la Lorraine. L'analyse porte sur 4 thèmes : les dépenses en santé, le cadre réglementaire et budgétaire, les résultats des entretiens, les éléments de réflexion autour des pratiques.

6 Cette partie s'appuie sur les travaux de Horwitz (2009) et de Toma (2012).

7 Ordonnance 2005-406 du 2 mai 2005, sur la nouvelle gouvernance hospitalière : rénovation de l'organisation hospitalière, instauration d'une nouvelle tarification à l'activité, gouvernance exercée par un comité exécutif.

8 (Martial, Naudy-Fesquet, Roosz et Trony, 2016).

9 Protection sociale à destination des personnes ayant de faibles ressources ; depuis 2016, la CMU est remplacée progressivement par la PUMA, Protection Universelle Maladie et par la CMU-C (CMU complémentaire)

10 Selon l'IRDES (Institut de Recherche et Documentation en Économie de la Santé) cela se traduit par des difficultés d'accès liées à l'absence de praticiens ou à leur éloignement, des délais de rendez-vous très importants chez les professionnels de santé présents (ce qui peut conduire certaines personnes à renoncer aux soins), des difficultés de permanence des soins durant les périodes ou les horaires de fermeture des cabinets médicaux, ce qui alourdit la fréquentation du SAMU (Service d'Aide Médicale Urgente) ou des services des urgences des hôpitaux pour des actes ou des pathologies qui ne relèvent pas de ce pourquoi ils ont été créés

11 Système d'information : ensemble organisé de ressources (matériels, logiciels, processus, personnel, données) permettant de collecter, structurer, stocker, analyser, et diffuser de l'information dans une organisation à des fins de décision

12 Association «Autrement, pour un autre regard sur son poids », Santé publique France : établissement public administratif sous tutelle du ministre chargé de la Santé créé par le décret n 2016-523 du 27 avril 2016 ; il fait partie de la loi de modernisation du système de santé (loi n 2016-41 du 26 janvier 2016).

13 EHESP (École des Hautes Études en Santé Publique) : établissement de formation et de recherche en santé publique et action sociale. Elle forme les cadres supérieurs de fonction publique dans le domaine de la santé et affirme avoir «l'ambition de nourrir un dialogue fructueux entre santé publique et management».

14 Anti-diabétique prescrit pendant plus de 30 ans à 5 millions de personnes en France, largement utilisé comme coupe-faim et responsable de plusieurs centaines de mort. Il a été retiré du marché en 2009, après les révélations du Dr Irène Frachon.

15 Médicament générique d'un anti-cancéreux (pour traiter le cancer du sein) ayant entraîné le décès de plusieurs patientes à la suite d'un choc septique entre 1996 et 2016. Une enquête de l'ASNM est en cours. 


\section{RÉFÉRENCES}

Benzerafa Alilat, M., Garcin L. et Gibert P. (2016). Le volet performance de la LOLF. Standardisation et résilience d'un genre entre rationalité politique et rationalité de gestion. Revue française de gestion. 7(260), 11-31. doi: 10.3166/rfg.2016.00063

Clement, J.-M. (2009). La nouvelle loi Hôpital, patients, santé, territoire. Bordeaux, France : Les Études Hospitalières.

Horwitz, M. (2009). L'avenir de la santé. Paris, France : Colin.

Laude, A. et Tabuteau D. (2016). Loi santé, regards sur la modernisation de notre système de santé. Rennes, France : Presses de l'École des hautes études en santé publique.

Martial, F., Naudy-Fesquet, I., Roosz, P. et Tronyo, J. (2016). Tableaux de l'économie française. Paris, France : Institut national de la statistique et des études économiques.

Toma, O. (2012). Hippocrate au secours! Comment bâtir un système de santé durable? Paris, France : Pearson. 